\title{
VIRTUAL TESTING INTEGRATION AND MATERIAL ALLOWABLES GENERATION
}

\author{
S. Miot ${ }^{1^{*}}$, L. Barrière ${ }^{1}$, J. Camacho Casero $^{1}$ and M. De Lozzo ${ }^{1}$ \\ ${ }^{1}$ IRT Saint Exupéry, Materials Dpt, Toulouse, France \\ *stephanie.miot@irt-saintexupery.com
}

Reducing uncertainties and therefore risks in structural design implies determining accurate statistically-based properties of the material. IRT Saint-Exupéry has been developing a software solution called VIMS that uses the GEMS open source python library [1] to generate material allowables. VIMS offers a framework to integrate, evaluate and use advanced composites models in association with experimental data post-processing, decision-making support and an innovation-friendly environment that facilitates the deployment within design offices. Fig. 1 illustrates the strategies implemented in VIMS.

Models developed by Onera [2], University of Porto and University of Girona [3] have been wrapped into VIMS. Toolboxes are provided to facilitate model exploration through sensitivity analyses and surrogate model building. An automated workflow was implemented to assist the user in the calibration phase, i.e. the identification of the model parameters from standard tests. The model validation phase provides the user with error maps and guidance on the complementary experimental tests that could improve the confidence in the model. The generation of B-basis strength values can be based on different methods including the Composite Materials Handbook-17 (CMH-17) method [4] and a new efficient UQ\&M methodology based on adaptive surrogate models and active learning techniques.

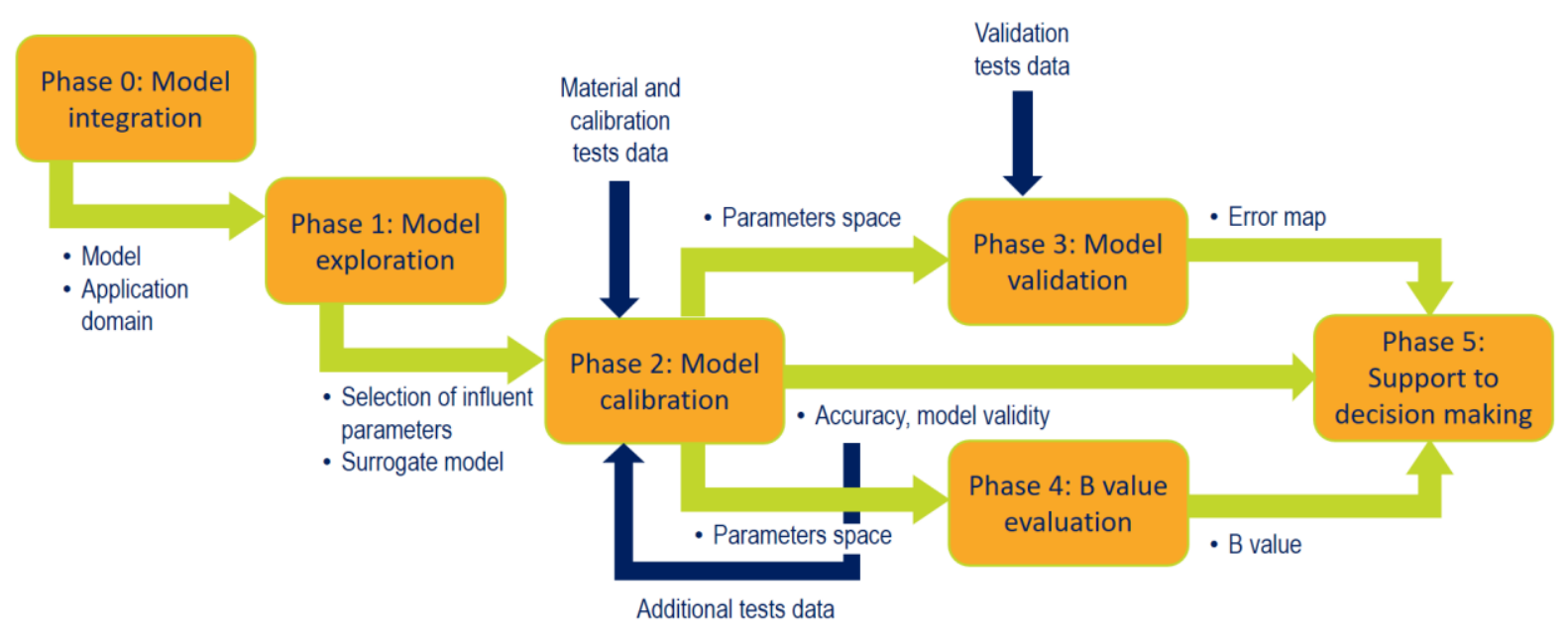

Figure 1. VIMS - Virtual testing Integration for decision Making Support

\section{References}

[1] F. Gallard, GEMS: a Python library for automation of multidisciplinary design optimization process generation. In SCITECH Forum, AIAA/ASCE/AHS/ASC Str., Str. Dyn. \& Mat. Conf., Kissimmee, USA, Jan. 2018.

[2] F. Laurin, et al., A multiscale hybrid approach for damage and final failure predictions of composite structures, Journal of Composite Materials, vol.47(20-21), pp.2713-2747, 2013.

[3] E.V. González, et al., Simulation of drop-weight impact and compression after impact tests on composite laminates. Composite Structures, vol.94(11), pp.3364-3378, 2012.

[4] U. S. D. of Defense., Military Handbook-MIL-HDBK-17-1F: Composite Materials Handbook, Volume 1, US Department of Defense, 2002. 